$15^{\text {th }}$ International Conference on

AEROSPACE SCIENCES \& AVIATION TECHNOLOGY,

$\boldsymbol{A S A T}$ - 15 - May 28 - 30, 2013, Email: asat@mtc.edu.eg,

Military Technical College, Kobry Elkobbah, Cairo, Egypt,

Tel: +(202) 24025292 -24036138, Fax: +(202) 22621908

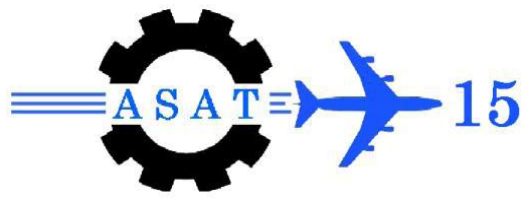

\title{
Remote Sensing Images Fusion Technique Based on ICA and IHS.
}

\author{
A. El Ejaily ${ }^{*}$, F. Eltohamy ${ }^{\dagger}$, M.Y. EL Nahas ${ }^{\ddagger}$, G. Ismail $^{\dagger}$
}

\begin{abstract}
Image fusion is a technique of combining complementary information from multiple images originated from single or different sources to form single fused image. This fused image has better quality compared to the original input images. In this paper a new fusion technique based on independent component analysis (ICA) and IHS transformation is proposed. A comparison of this new technique with PCA, IHS, and ICA-based fusion techniques is given. Ikonos-2 data are used to test the new and other three techniques.
\end{abstract}

Four evaluation criteria are used for quantitative assessment the quality of the obtained fused images. To assess the spectral quality the spectral discrepancy $\left(D_{k}\right)$ and correlation coefficients $(C C)$ are used. For spatial quality assessment high pass correlation coefficients $(H P C C)$ and information entropy $(E N)$ are used. The results of the new technique show higher performance compared to the other techniques.

Keywords: Image fusion; principal component analysis (PCA); independent component analysis (ICA); intensity hue saturation (IHS); remote sensing images.

\section{Introduction}

Image fusion is the process of combining relevant information from two or more images into a single image. The resulting image will be more informative than any of the input images.

With the availability of multisensor data in many disparate fields, such as remote sensing, machine vision, robotics, medical images, and military applications, multisensor data fusion has emerged as a new and promising research area [1].

In remote sensing applications, the increasing availability of space borne sensors gives a motivation for different image fusion algorithms. Several situations in image processing require high spatial and high spectral resolution in a single image [1].

Many techniques and software tools for fusing images have been developed [3-5]. From the well-known methods, are the Brovey method, the intensity-hue-saturation (IHS), principal component analysis (PCA), and discrete wavelet transform (DWT). Although these methods provide fused images with high spatial resolution, but they suffer from spectral distortion that introduced during the fusion process.

Libyan armed forces, Libya

$\dagger$ Egyptian Armed Forces, Egypt.

* Alazhar University, Cairo, Egypt. 
The invention of multi-resolution analysis tool like wavelet transform allow to further improve the quality of the fused image, but it needs a proper selection of decomposed level according to the ratio between the PAN and MS images.

The former studies show that we can use independent component analysis (ICA) to achieve a fused image with a high spatial resolution and minimum spectral distortion $[6,7]$.

The ICA-based remote sensing image fusion is performed by transform the MS image into its independent components then the most significant IC is replaced by PAN image and then an inverse ICA is performed on the new combination to retain the fused image. The author in [8] used wavelet transform to improve the performance of ICA-fusion technique.

In this paper we propose a new technique based on the use of IHS transform and ICA to improve the ICA performance in the fusion of remote sensing images. First the MS and PAN images are fused using a classical ICA method to get intermediate fused image then we use IHS transform to transform the resultant fused image together with the original MS image to IHS color space, then in the last step inverse IHS is applied to color components - H \& S- of MS image and the intensity component of the intermediate fused image.

\section{Concept of Independent Component Analysis}

ICA is a computational method for separating a multivariate signal into additive subcomponents supposing the mutual statistical independence of the non-Gaussian source signals [9].

The estimation of the data model of independent component analysis is usually performed by formulating an objective function and then minimizing or maximizing it. Therefore, the properties of the ICA method depend on both the objective function and the optimization algorithm. Assume that there is an M-dimensional zero mean vector $S=\left(S_{1}, S_{2}, \ldots, S_{M}\right)^{T}$, whose components are mutually independent distribution. The vector $\mathrm{S}(\mathrm{t})$ corresponds to $\mathrm{n}$ independent scalar valued source signal $S_{i}$. we can write the multivariate p.d.f of the vector as the product of the marginal independent distributions:

$$
p(s)=\prod_{i}^{M} p_{i}\left(S_{i}\right)
$$

a data vector $x=\left(x_{1}, x_{2}, \ldots, x_{N}\right)^{T}$ is observed vector at point $\mathrm{t}$, such that

$$
s(t)=A x(t)
$$

where, $\mathrm{A}$ is an $\mathrm{N}^{*} \mathrm{M}$ scalar matrix which is called mixing matrix.

The goal of ICA is to find a linear transformation $W$ of the correlative signal $x$ that makes the outputs as independent as possible:

$$
u(t)=W x(t)=W A^{-1} s(t)
$$

where, $\mathrm{u}$ is an estimate of the sources. 


\section{Image Fusion Technique Based on ICA-IHS}

Although the main significant IC contains the main content of color image, the spectral and spatial information of the color image is not completely separated. Some of spatial information are also located in the other ICs. Therefore, the ICA- based fusion method tends to produce a fused image with high spatial detail, but also may lead to spectral distortion in some local regions. To minimize this spectral distortion we use IHS transform to further process the resultant fused image and replacing its color components with that of original MS image.

This is the central idea of the improved ICA fusion method. Figure 1 outlines the general procedure to fuse MS and PAN images using the improved ICA method based on IHS, and the detailed steps of ICA-IHS fusion method are as follows:

1) Registering the MS and the PAN images with the error pixel.

2) Applying ICA to MS bands to transform the correlated band to a linear combination of independent components.

3) The most significant component of step (2) is replaced with PAN image, then the intermediate fused image is obtained by inverse ICA.

4) To overcome the spectral distortion of the intermediate fused image, we transform this fused image and the original MS image to IHS color space and we replace the color components (hue \& saturation) of the intermediate fused image with that of original MS image

5) At last the final fused image is obtained by inverse IHS of the new combination of step (4).

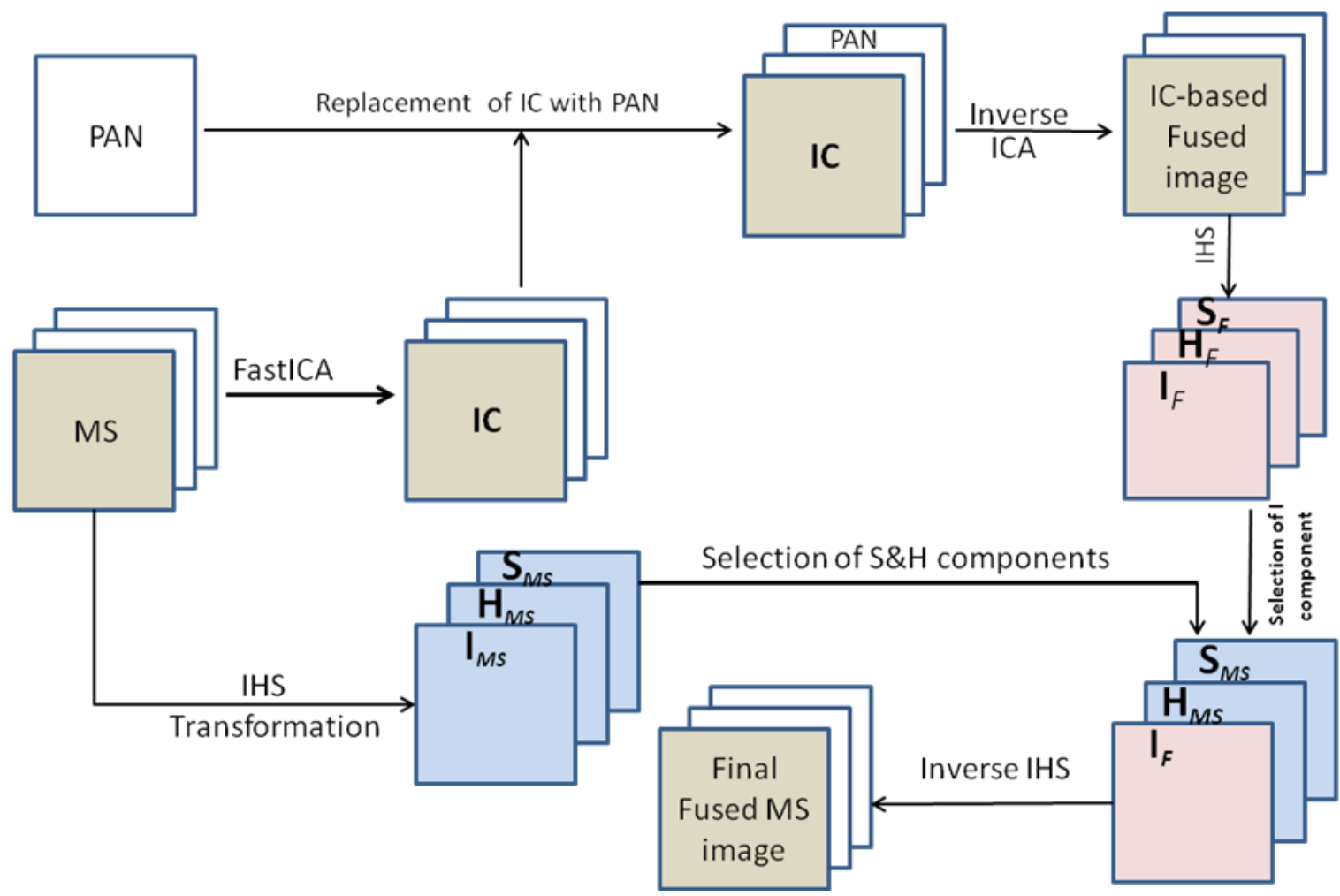

Fig. 1 Flow diagram of the proposed image fusion technique 


\section{Quality Assessment Criteria}

Quality refers to both the spatial and spectral quality of images [10]. The goal of image fusion is to increase the spatial resolution of the MS images while preserving their spectral contents. In this paper we use correlation and discrepancy to assess the spectral quality of the fused image, while for the measurement of spatial quality the high-pass correlation and entropy are used.

\section{A. Spectral quality assessment}

For a good spectral fidelity of the fused image, the low spatial frequency information in the high-resolution image shouldn't be transferred to the fused image[11]. The following measures are used to test the spectral quality of the fused image:

Correlation coefficient: $\mathbf{C C}$ measures the correlation between the original MS image and the fused images. The higher the correlation between the fused and the original images, the better the estimation of the spectral values. The ideal value of correlation coefficient is 1 .

The formula to compute the correlation between two images A\&B is given by [12]:

$$
\operatorname{Corr}(A / B)=\frac{\sum_{i=1}^{N} \sum_{j=1}^{M}\left(A_{i, j}-\bar{A}\right)\left(B_{i, j}-\bar{B}\right)}{\sqrt{\sum_{i=1}^{N} \sum_{j=1}^{M}\left(A_{i, j}-\bar{A}\right) \sum_{i=1}^{N} \sum_{j=1}^{M}\left(B_{i, j}-\bar{B}\right)}}
$$

where $\bar{A}$ and $\bar{B}$ are the mean values of the corresponding data set, (i,j) denotes a given pixel, $\mathrm{N} \& \mathrm{M}$ are the image dimension.

Discrepancy: The spectral quality is measured by the discrepancy $D_{k}$, at each band as follows [13]:

$$
D_{k}=\frac{1}{M * N} \sum_{i=1}^{M} \sum_{j=1}^{N}\left|F_{K_{i, i} j}-M S_{K_{i}, i j}\right| ; K=R, G, B
$$

where $F_{K_{i, j}, j}$ and $M S_{K_{i}, j}$ are the pixel values at position $(i, j)$ in the $K^{\text {th }}$ band of the fused and original MS image, respectively. It is known that the spectral quality of the image increases as $D K$ decreases.

\section{B. Spatial quality assessment}

For a good spatial fidelity of the fused image, the high spatial frequency information in the high-resolution image(that represent image edges) should be transferred to the fused image. The indexes which can reflect the spatial fidelity of fused image include:

High pass correlation: The spatial quality is obtained by computing the correlation coefficient, ( $H P C C)$, between the high pass component of the fused image bands and the original PAN image. The high pass component is used in the evaluation of this criterion because the spatial information is mostly concentrated in the high frequency domain. The spatial quality of the image is directly proportional to HPCC. To extract the high frequency data we apply the following convolution mask to the images [14]:

$$
\operatorname{mask}=\left|\begin{array}{ccc}
-1 & -1 & -1 \\
-1 & 8 & -1 \\
-1 & -1 & -1
\end{array}\right|
$$


Entropy: The Entropy (EN) can show the average information included in the image and reflect the detail information of the fused image. The greater the Entropy of the fused image is, the more abundant information included in it, and the greater the quality of the fusion is.

According to the information theory of Shannon, The image entropy is given by [12]:

$$
E N=-\sum_{g=0}^{L-1} p(g) \log _{2} p(g)
$$

where $\mathrm{p}(\mathrm{g})$ is the probability of grey level $\mathrm{g}$, and the range of $\mathrm{g}$ is $[0, \ldots, \mathrm{L}-1]$. $\mathrm{L}$ is the maximum value of the gray levels.

\section{Experimental Results and Discussion}

The Ikonos-2 data were selected for carrying out the experimental work of this study, which were acquired on 22 of Sep 2012. 09:50 GMT. The site were located in Tripoli, Libya. The images are composed of MS image with spatial resolution of $4 \mathrm{~m}$ and PAN image with spatial resolution of $1 \mathrm{~m}$. Sub-images of $1000 \times 1000$ pixels have been considered. Figure 2(a) and figure 2(b) shows the MS and PAN images respectively.

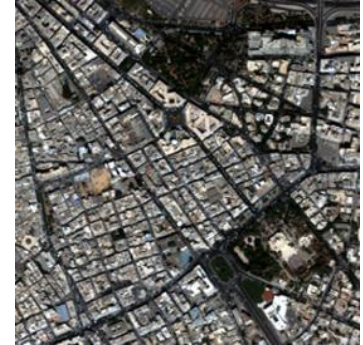

(a)

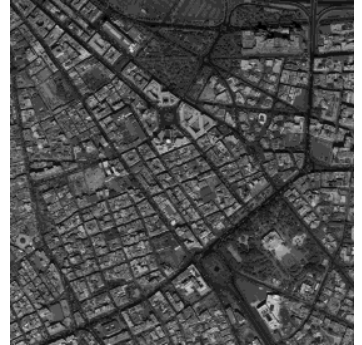

(b)

Fig 2. Ikonos data of Tripoli (a) MS image ; (b) PAN image

In order to examine the performance of the proposed image fusion method, we compare it with other fusion methods like IHS, PCA, and ICA. The resultant fused images are shown in figure 3 .

The visual inspection of the obtained fused images shows that all fusion methods retained an acceptable amount of spatial detail; the PCA method preserves fewer details than IHS. We get a maximum spatial detail in the fused image when we use ICA, but the distortion of the original color is very obvious. The improved ICA method proposed in this paper can solve this problem effectively, and we can achieve a fused image with a better balance between spectral characteristic preservation and high spatial resolution

To objectively assist the spatial quality of the obtained fused images we measure the highpass correlation coefficients $(H P C C)$ and the information entropy $(E N)$, the results of those two measures are shown in table 1, the results show that a fused image with maximum spatial details is achieved when we used the proposed method. To assess the spectral quality we use the measurements of spectral discrepancy $\left(D_{k}\right)$ and correlation coefficients (CCs). Table 2 shows the results which indicate that the improved ICA and IHS methods have almost the same spectral quality better than the other fusion techniques. 


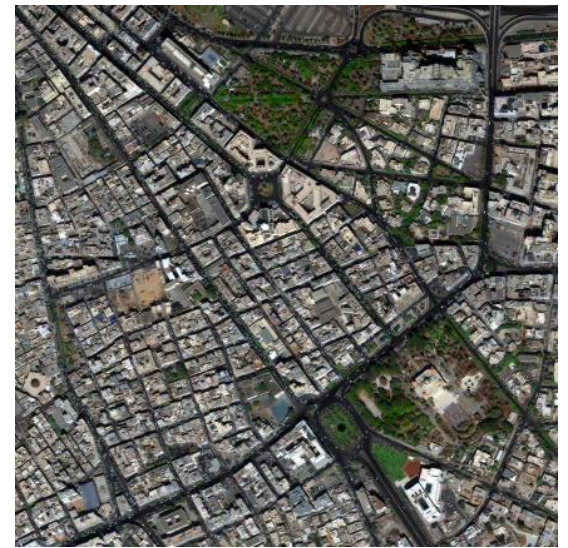

(a)

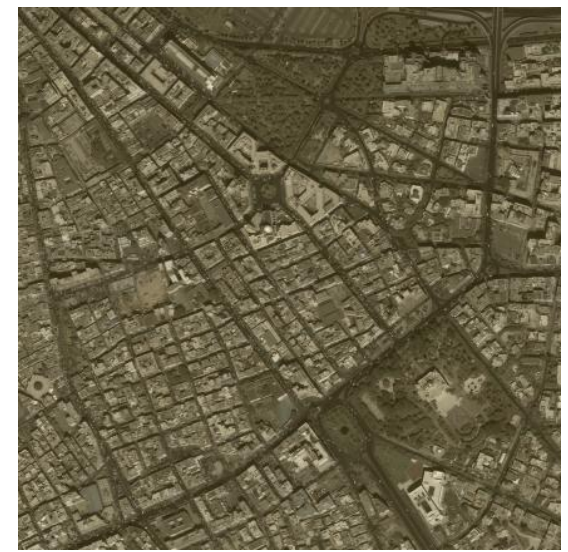

(c)

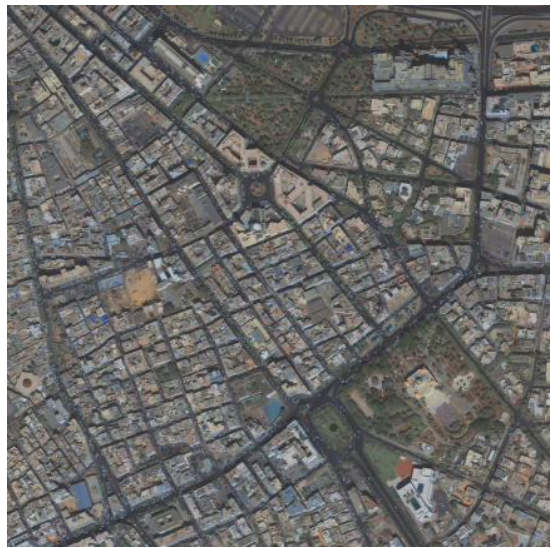

(b)

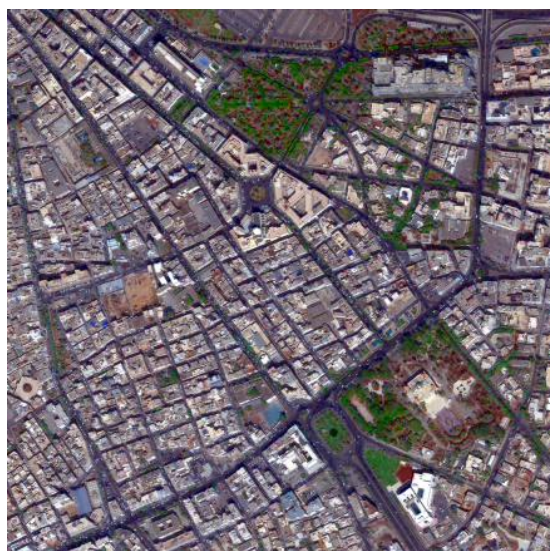

(d)

Fig. 3 The results of different fusion methods using Ikonos data (a) fused image by IHS ; (b) fused image by PCA; (c) fused image by ICA; (d) the fusion result of applying the proposed method.

Table 1 The results of spatial quality assessment

\begin{tabular}{l|c|c|c|c|c|c}
\hline \hline \multirow{2}{*}{ Fusion method } & \multicolumn{3}{|c|}{ HPCC } & \multicolumn{3}{c}{$E N$} \\
\cline { 2 - 7 } & $\mathrm{R}$ & $\mathrm{G}$ & $\mathrm{B}$ & $\mathrm{R}$ & $\mathrm{G}$ & $\mathrm{B}$ \\
\hline HIS & 0.9214 & 0.9262 & 0.9305 & 7.6868 & 7.725 & 7.6815 \\
\hline PCA & 0.9697 & 0.9668 & 0.9603 & 7.0767 & 7.0021 & 6.9618 \\
\hline ICA & 0.9853 & 0.9795 & 0.9678 & 7.0113 & 6.9662 & 6.8965 \\
\hline Proposed method & 0.9536 & 0.9486 & 0.9614 & 7.6914 & 7.6787 & 7.5961 \\
\hline \hline
\end{tabular}

Table 2 The results of spectral quality assessment

\begin{tabular}{l|c|c|l|l|l|l}
\hline \hline \multirow{2}{*}{ Fusion method } & \multicolumn{3}{|c|}{$C C$} & \multicolumn{1}{c}{$D_{k}$} \\
\cline { 2 - 7 } & $\mathrm{R}$ & $\mathrm{G}$ & $\mathrm{B}$ & $\mathrm{R}$ & \multicolumn{1}{c}{$\mathrm{G}$} & $\mathrm{B}$ \\
\hline HIS & 0.9109 & 0.9063 & 0.9074 & 0.138 & 0.1405 & 0.1312 \\
\hline PCA & 0.9112 & 0.9116 & 0.9105 & 0.1484 & 0.146 & 0.1436 \\
\hline ICA & 0.9094 & 0.9069 & 0.8492 & 0.1482 & 0.1431 & 0.1561 \\
\hline Proposed method & 0.9221 & 0.9156 & 0.9177 & 0.1439 & 0.139 & 0.1445 \\
\hline \hline
\end{tabular}




\section{Conclusion}

In this paper we present an improved ICA image fusion method. In the convenient way of ICA-based image fusion, the most significant IC is replaced with PAN image then the fused image is obtained through an inverse ICA. This fused image is further processed by transform it to IHS color space. Then we select the intensity component together with color components ( $\mathrm{H}$ and $\mathrm{S}$ ) of the original MS image to produce a new fused image using inverse IHS transformation. The new fused image will contain the original color with a high spatial detail. The QuickBird images were used to show the performance of the proposed method. The results show that the proposed method can preserve abundant spectral information and higher spatial resolution, and has a better performance compared with other techniques.

\section{References}

[1] R.S. Blum, Z.Liu "Multi-Sensor Image Fusion and Its Application", New York, Taylor\&Francis Group, 2006.

[2] C.Pohl, J.L. Van Genderen "Multisensory image fusion in remote sensing: concepts, methods and applications" int. j. remote sensing, , vol. 19, no. 5, pp823-854,1998.

[3] María González-Audícana, José Luis Saleta, Raquel García Catalán, and Rafael García, "Fusion of Multispectral and Panchromatic Images Using Improved IHS and PCA Mergers Based on Wavelet Decomposition", IEEE Transaction on Geoscience and Remote Sensing, vol. 42, No. 6, June 2004.

[4] Myungjin Choi, "A New Intensity-Hue-Saturation Fusion Approach to Image Fusion With a Tradeoff Parameter", IEEE Transaction on Geoscience and Remote Sensing, vol. 44, No.6, June 2006.

[5] Li S., Kwok J.T., and Wang Y., "Using the Discrete Wavelet Transform frame transform to merge Landsat TM and SPOT panchromatic images", Information Fusion 3, pp. 17-23 2002.

[6] Hongzhi Wang, Lu wei, Cai he, "An adaptive image fusion algorithm based on 1CA", International Conference on Computer. Mechatronics, Control and electronic engineering(CMCE) 2010.

[7] Meng Wang, Jian Yang, "Multi-sensor Image Fusion with ICA Based and Region Rule", $10^{\text {th }}$ Intl. Conf. on Control, Automation, Robotics and Vision, December 2008.

[8] F. Chen, F. Qin, G. Peng, S. Chen, "Fusion of Remote Sensing Images Using Improved ICA Mergers Based on Wavelet Decomposition", International Workshop on Information and Electronic Engineering (IWIEE). 2012.

[9] A. Hyvarinen, J. Karhunen, E. Oja, "Independent Component Analysis", New York: John Wiely \& Son, Inc; 2001.

[10] L. Wald, T. Ranchin, M. Mangolinii, "Fusion of Satellite Images of Different Spatial Resolution: Assessing the Quality of Resulting Images", Photogrammetric Engineering \& RemoteSensing, Vol. 63, No. 6, pp. 691-699, 1997.

[11] M. FallahmYakhdani, A.Azizi, "Quality Assessment of Image Fusion Techniques for Multisensor High Resolution Satellite Images( Case Study: IRS-P5 and IRS-P6 Satellite Images)”, ISPRS TC Symposium, Vol XXXVIII, PART 7b July 5-7, 2010.

[12] S.S.Han, H.T.Li, H.Y. Gu, "The Study of Image Fusion for High spatial Resolution Remote Sensing Images", The international Archives of the Photogrammetry, Remote sensing and Spatial Information Scinces. Vol. XXXVII. part B7. Beijing 2008.

[13] Zhenhua Li, Z. Jing, X. Yang, "Color Transfer Based Remote Sensing Image Fusion using Non-Separable Wavelet Frame Transform”, Pattern Recognition Letters, vol. 26 Issue. 13, pp. 2006-2014, 2005.

[14] Zhou, D.L.Civco. J. A. Silander, “A Wavelet Transform Method to Merge Landsat TM and Spot Panchromatic Data”, Int. J . Remote Sensing Vol. 19, No. 4, 743- 757, 1998. 